\title{
Is Primary Repair of Tracheobronchial Rupture Curative?
}

\author{
Abdel-Maged Salem, Alaa Brik*, Ali Refat, Karem Elfagharany, Abdalla Badr \\ Department of Cardiothoracic Surgery, Zagazig University Hospital, Zagazig, Egypt. \\ Email: *alaabrik@yahoo.com \\ Received January $21^{\text {st }}, 2013$; revised February $26^{\text {th }}, 2013$; accepted March $9^{\text {th }}, 2013$ \\ Copyright (C) 2013 Abdel-Maged Salem et al. This is an open access article distributed under the Creative Commons Attribution Li- \\ cense, which permits unrestricted use, distribution, and reproduction in any medium, provided the original work is properly cited.
}

\begin{abstract}
Background: Tracheobronchial disruption as a result of blunt thoracic trauma is a rare entity and only clinically serious lesions come to our notice, which can be life-threatening and need prompt recognition and treatment. Objectives: To review the authors' experience with tracheobronchial injuries to emphasize the need for prompt diagnosis and treatment to avoid lethal complications including severe hypoxic organ failure, sepsis, mediastinitis and bronchopleural fistula. Patients and methods: A retrospective study of total 32 patients with tracheobronchial injury from 2001 to 2011. This study limited to patients with thoracic tracheal or bronchial injury, excluding those with cervical injuries. The study includes collected information about mechanism of injury, presentation, time until diagnosis and treatment, anatomical site of injury, type of treatment, diagnostic methods, duration of follow up and outcome. Results: Twenty-four patients were male (75\%) and eight were females (25\%). Patient's ages ranged from 7 - 53 years. Majority of cases was referred because of blunt trauma in 15 cases (46.8\%), 6 (18.75) motor vehicle accident, 5 (15.6\%) fall from a height and 4 (12.5\%) with trauma by heavy object, while 8 cases (25\%) were referred due to penetrating injury and 2 cases (6.25\%) due to iatrogenic injury. In initially diagnosed group, the predominant clinical signs that give a suspicion of tracheobronchial disruption were increased subcutaneous surgical emphysema, shortness of breath, hemoptysis. After the admission to emergency unit, all of them were examined radiologically by chest X-ray film. Longitudinal tear of right upper lobe bronchus was found in 8 cases (32\%), complete cut of right upper lobe bronchus in 4 cases (16\%), tear of right intermediate bronchus in 4 cases (16\%), 3 cases with clear cut left upper lobe (12\%), longitudinal tear of distal lateral tracheal wall extend to right upper lobe in 2 cases (8\%), 2 cases (8\%) showed complex disruption of distal trachea right main with carinal tear and 2 cases (8\%) with longitudinal tear of membranous wall of the trachea. 17 patients from early diagnosed cases had concomitant comorbid extra thoracic injuries at the time of diagnosis in the form of abdominal trauma in 12 cases, skeletal fractures in 9 cases and head injury in 5 cases. Conclusion: In a patient with a complex bronchial rupture, primary repair of the bronchus can be possible with complete functional preservation of the lung tissue.
\end{abstract}

Keywords: Tracheobronchial Disruption; Trauma

\section{Introduction}

Tracheobronchial disruption, because of blunt thoracic trauma, is a rare entity and only clinically serious lesions come to our notice which can be life-threatening and need prompt recognition and treatment [1]. In Bertelsen and Howitz [2]'s study, approximately 3\% of 1128 patients at autopsy had evidence of Tracheobronchial injury, with most (81\%) having died before ER presentation. Many patients die before arrival to the hospital. Some of them are misdiagnosed with multiple injuries.

The purpose of the present article was to review the authors' experience with tracheobronchial injuries to em-

\footnotetext{
"Corresponding author.
}

phasize the need for prompt diagnosis and treatment to avoid lethal complications including severe hypoxic organ failure, sepsis, mediastinitis and bronchopleural fistula.

\section{Materials and Methods}

Collection of patient's data was obtained by reviewing the operative files from 2001 to 2011 at Cardiothoracic Surgery Department, Zagazig University (Emergency Unit) for this retrospective study. Our cardiothoracic emergency unit serve large area in Egypt receiving nearly 3000 - 5000 patients per year with problems related to cardiothoracic surgery mainly Trauma either penetrating or blunt trauma. A total of 32 patients with tracheobron- 
chial injury were gathered and enrolled in this retrospective study. This study limited to patients with thoracic tracheal or bronchial injury, excluding those with cervical injuries The study include collected information about mechanism of injury, presentation, time until diagnosis and treatment, anatomical site of injury, type of treatment, diagnostic methods, duration of follow up and outcome.

In our series, all patients in both early and delayed diagnosis patients underwent posterolateral thoracotomy exposure. Ventilation maintained by single lumen end tracheal tube that pushed guided by surgeon to patent healthy bronchus away from the bronchial injury allowing selective ventilation to the healthy airway and protecting the normal site from aspiration. For complete transacted bronchus repair depend on ventilation of healthy bronchus after closure of the proximal stump by pice of gauze to prevent ventilation leakage during repair. Both proximal and distal bronchial stump were exposed for and end to end anastomosis.

After exposure of the bronchial tear as illustrated in Figure 1 which in one of our cases of left main bronchus disruption with irregular edges, repair was done using vicryl (3/0) for all cases.

Intercostals muscle flap was used to strengths the repair in 12 cases, plural flap for wrapping the suture line after completion in 8 cases and in 5 cases pericardial strip taken for wrapping the suture line fixed from periphery by $5 / 0$ proline suture. In delayed diagnosed cases (7) resection done in one case (right upper lobectomy because of massive fibrotic bronchial ends and destroyed lobe. The rest 6 cases end-to-endanastamosis after refreshing the edges of transected bronchus in all of them pleural flap used to cover the anastomotic site. In one case of them, failure to expansion lung which necessitate multiple flexible bronchoscope for aspiration of secretion in the first week of surgery finally pass postoperatively with out complication. All case examined after one month

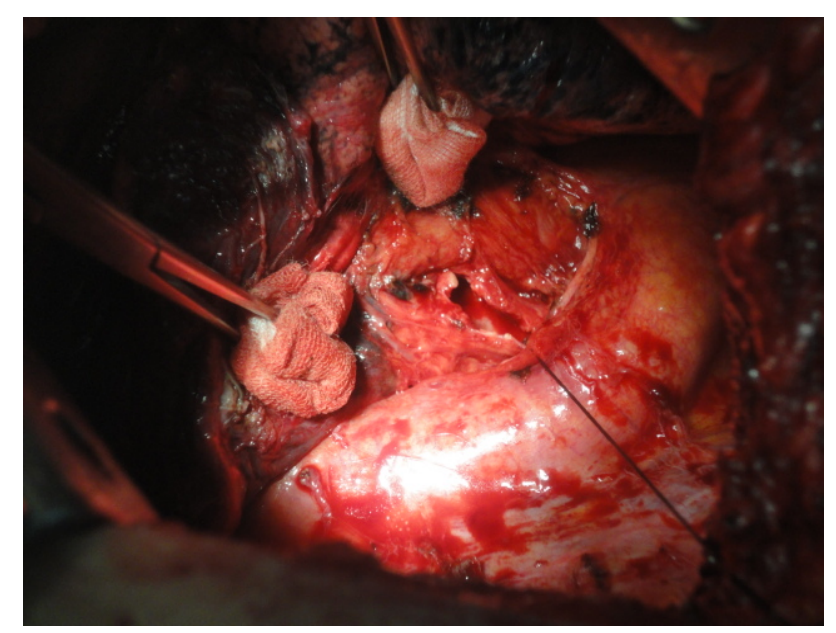

Figure 1. It shows rupture left main bronchus. by bronchoscope; all of them showed patent bronchus except one case which shows narrowed stricture and follow up bronchoscope for aspiration of secretion was done and the patient remained asymptomatic.

Statistical analysis: Results were analyzed using SPSS version 15.0 (Statistical Package for Social Science, SPSS Inc., Chicago, IL, USA). Continuous variables, such as age were expressed as the mean \pm S.D. Categorical variables were expressed by number (n) and frequencies (\%).

\section{Results}

All injuries involved the intrathoracic trachea with 32 cases as both acute and delayed cases has different epidemiology, management plane and subsequently in there outcome which we categorized according to time or presentation into acute 25 (Group A) and delayed 7 (Group B). Out of these patients, twenty four patients were male (75\%) and eight were females (25\%). Patient's ages ranged from 7 - 53 years. Majority of cases were referred because of blunt trauma in 15 cases (46.8\%), 6 (18.75) motor vehicle accident, 5 (15.6\%) fall from a height and 4 (12.5\%) with trauma by heavy object, while 8 cases (25\%) were referred due to penetrating injury and 2 cases (6.25\%) due to iatrogenic injury (Table 1). In initially diagnosed group (25 cases), the predominant clinical signs that give a suspicion of tracheobronchial disruption were pneumothotax $(78.1 \%)$ increased subcutaneous surgical emphysema (21, 84\%), shortness of breath $(22,88 \%)$ and hemoptysis $(9,36 \%)$, as shown in Table 2. After admission to emergency unit, all of them were examinedradiologically by chest X-ray film, Persistent massive air leak from intercostals tube that was inserted in cases with radiological signs of pneumothorax, pneumomediastinum and surgical emphysema establish the diagnosis in 16 patients (64\%), especially with increasing dyspnea and cyanosis which necessitate emergency thoracotomy without additional investigation. In 6 cases (24\%), rigid bronchoscope was carried out and was helpful in diagnosis of injury (bronchial tears). The cases with hemothorax were managed initially by ICT followed by emergency thoracotomy

Table 1. Mechanism of injury in initially diagnosed patients.

\begin{tabular}{ccc}
\hline & No & \% \\
\hline Blunt trauma & 15 & 46.8 \\
Motor vehicle accident & 6 & 18.75 \\
Fall from a height & 5 & 15.6 \\
Trauma by heavy & 4 & 12.5 \\
object & & 25 \\
Penetrating injury & 8 & 6.25 \\
Iatrogenic injury & 2 & \\
\hline
\end{tabular}


Table 2. Clinical findings in 32 cases.

\begin{tabular}{ccc}
\hline & No & \% \\
\hline $\begin{array}{c}\text { Subcutaneous surgical } \\
\text { emphysema }\end{array}$ & 21 & 75 \\
Shortness of breath & 22 & 79.2 \\
Hemoptysis & 9 & 28.1 \\
Atelecatsis/collapse & 8 & 25 \\
Pneumothorax & 25 & 78.1 \\
\hline
\end{tabular}

because of massive drain from chest tube. In the initially diagnosed cases; either those diagnosed clinically or by bronchoscope data correlated with intraoperative finding found that most of lesions were detected on the right main bronchus and complete avulsion of the left main bronchus.

Longitudinal tear of right upper lobe bronchus was found in 8 cases (32\%), complete cut of right upper lobe bronchus in 4 cases (16\%), tear of right intermediate bronchus in 4 cases (16\%), 3 cases with clear cut left upper lobe (12\%), longitudinal tear of distal lateral tracheal wall extend to right upper lobe in 2 cases (8\%), 2 cases (8\%) showed complex disruption of distal trachea right main with carinal tear and 2 cases (8\%) with longitudinal tear of membranous wall of the trachea Table 3. 17 patients from early diagnosed cases (68\%) had concomitant comorbid extra thoracic injuries at time of diagnosis in the form of abdominal trauma in 12 cases, skeletal fractures in 9 cases and head injury in 5 cases. These injuries necessitate laporotomy in 8 cases, evacuation of extramural hematoma in one case and fixation of bone fracture (Table 4). On The other side, the seven cases in which diagnosis made lately, all of these cases were referred to us for bronchoscopic examination because of their clinical presentations by recurrent chest infection and with heomptysis and radiological findings of atelectasis and/or localized persistant lung collapse.In bronchoscopic evaluation the prominent finding was stricture and granulation tissue in 2 cases (28.57\%) which necessitate biopsy that found chronic non Specific inflammatory tissues, complete obstruction of main bronchi in 4 cases. In those cases, the bronchial injuries correlated with operative finding were sited on left main bronchus (3 cases), left upper lobe bronchus in one case, right upper lobe bronchus in 2 cases and right main bronchus in one case. Computed tomography was performed in all neglected case with no specific finding radiological and bronchoscope findings and history of chest trauma clarify the probability of bronchial in juries.

\section{Discussion}

Bronchial rupture due to the blunt thoracic trauma is rarely seen. Difficulties of the diagnosis may cause delay in the treatment. The initial assessment of major airway injuries involved the traditional ABCs of resuscitation as
Table 3. Site of bronchial injury in initially diagnosed group (25 cases).

\begin{tabular}{|c|c|c|}
\hline & No & $\%$ \\
\hline $\begin{array}{l}\text { Longitudinal tear of right } \\
\text { upper lobe bronchus }\end{array}$ & 8 & 32 \\
\hline $\begin{array}{l}\text { Complete cut of right upper } \\
\text { lobe bronchus }\end{array}$ & 4 & 16 \\
\hline $\begin{array}{l}\text { Tear of right intermediate } \\
\text { bronchus }\end{array}$ & 4 & 16 \\
\hline Clear cut left upper lobe & 3 & 12 \\
\hline $\begin{array}{l}\text { Longitudinal tear of distal } \\
\text { lateral tracheal wall extend to } \\
\text { right upper lobe bronchus }\end{array}$ & 2 & 8 \\
\hline complex inury at the carina & 2 & 8 \\
\hline $\begin{array}{c}\text { Membranous wall of the } \\
\text { trachea }\end{array}$ & 2 & 8 \\
\hline
\end{tabular}

Table 4. Associated injuries.

\begin{tabular}{ccc}
\hline & No & \% \\
\hline Abdominal trauma & 12 & 48 \\
Skeletal fractures & 9 & 36 \\
Head injury & 5 & 20 \\
\hline
\end{tabular}

outlined in the advanced trauma life support guideline [3]. Patients with tracheobronchial require urgent control of the airway, evacuation of blood and air from the pleural space by intercostal drainage. Orotracheal intubation was the most frequent method to control the airway. The pathogenesis of tracheobronchial rupture in blunt trauma can be divided into three mechanisms $[4,5]$ :

1) A traction is exerted on the carina;

2) The trachea and main bronchi are violently compressed while the glottis is closed;

3) A rapid deceleration may result in shearing forces at the posterior areas of fixation.

The constellation of persistent pneumothorax, massive air leak, and atelectatic lung in the presence of a well placed, functional thoracostomy tube constitues the "fallen lung" sign and is a chest roentgenogram finding of high specificity for intrathoracic airway rupture. A pneumothorax is frequently present but may be absent if the mediastinal pleura remains intact. For the diagnosis, bronchoscopy is the first method of choice [6].

It is reported that three-dimentional helical computed tomography might be used in the diagnosis [7]. Thoracic emphysema, respiratory distress, cough, hoarseness, hemoptysis and even cyanosis are the most consistent clinical findings in tracheal injury caused by non-penetrating blunt trauma; subcutaneous emphysema is the result of decompression of pneumomediastinum. This sign is nonspecific finding and is more commonly found in acute traumatized patients after difficult intubations, 
multiple rib fractures and penetrating chest wounds. Subcutaneous emphysema, pneumthorax, pneumomediastinum and air surrounding the main bronchi are the most radiological findings [7]. In blunt trauma lesions of the main bronchus are common. In all patients of our series, lesions were located at the main bronchus solely or concomitant with carina or lower tracheal rupture [8]. Lynn and Lyengar [9] reported that more than $80 \%$ of tracheobronchial injuries due to blunt trauma are located within $2.5 \mathrm{~cm}$ of the carina. Immediate primary repair of the bronchial rupture is advocated to preserve functional lung tissue and to provide the best long-term results. Even in complete ruptures, reanastomosis chance must be tried, even if the rupture is old $[10,11]$. As seen in our serious missing and delaying the diagnosis and surgical intervention of tracheobronchial disruption may be complicated by partial or complete airway stenosis need for resection. Given the good outcome of primary repair in our patient, we believe that close observation, prompt diagnosis, and correct surgical repair as described here is indispensable for both physician and surgeon in managing emergency patients. In cases where an early diagnosis of bronchial tear is not made and repair not done, one of the two clinical courses may follow:

1) An incomplete laceration heals with stricture formation and results in recurrent atelectasis, infection and bronchiectasis. This may require resection or bronchoplastic procedure;

2) When complete transection occurs, both ends of the severed bronchus granulate and heal. The distal bronchial tree fills with mucus, and the lung collapses. Infection does not occur and the parenchyma of the lung remains undamaged.

Therefore, the prognosis is better. The prognosis of tracheobronchial ruptures is often related to the severity of associated injuries in these multiple injured patients. Over the long term, bronchial stenosis by granulation tissue after primary repair of a bronchial rupture is to be feared. Regular bronchoscopic checks should be performed. Stenosis can be managed either with endoscopic therapy (electrocautery, laser cogulation, cryotherapy, dilatation and both silastic and metal stent placement) or, if endoscopic treatment is unsuccesful, by reoperation (sleeve or standard resection) [12].

\section{Conclusion}

In a patient with a complex bronchial rupture, primary repair of the bronchus can be possible with complete functional preservation of the lung tissue.

\section{REFERENCES}

[1] A. E. Flynn, A. N. Thomas and W. P. Schector, "Acute Tracheobronchial Injury,” The Journal of Trauma, Vol. 106, No. 1, 1994, pp. 74-78.

[2] S. Bertelsen and P. Howitz, "Injuries of the Trachea and Bronchi,” Thorax, Vol. 27, No. 2, 1972, pp. 188-194. doi:10.1136/thx.27.2.188

[3] H. Gaafar, M. R. Salama, G. O. Abu-Senna, A. Balbaa and S. H. El-Mallah, "Traumatic Injuries of the Bronchus," The Journal of the Egyptian Medical Association, Vol. 53, No. 1, 1970, pp. 13-28.

[4] R. B. Lee, "Traumatic Injury of the Cervicothoracic Trachea and Major Bronchi,” Chest Surgery Clinics of North America, Vol. 7, No. 2, 1997, pp. 285-304.

[5] A. Eijgelaar and J. N. Homan van der Heide, "A Reliable Early Symptom of Bronchial or Tracheal Rupture,” Thorax, Vol. 25, No. 1, 1970, pp. 120-125.

[6] D. E. Beesinger, F. L. Grover and J. K. Trinkle, “Tracheobronchialinjuries Secondary to Blunt Thoracic Trauma,” Texas MedClinic, Vol. 70, No. 4, 1974, pp. 74-77.

[7] F. Baumgarter, B. Sheppard, C. Virgilio, et al., "Tracheal and Main Bronchial Disruptions after Blunt Chest Trauma: Presentation and Management," The Annals of Thoracic Surgery, Vol. 50, No. 4, 1990, pp. 569-574. doi:10.1016/0003-4975(90)90191-8

[8] D. A. Kumpe, K. S. Oh and S. M. Wyman, "Characteristic Pulmonary Finding in Unilateral Complete Bronchial Transection,” American Journal of Roentgenology, Vol 110, No. 4, 1970, pp. 704-706.

[9] A. E. Lynn, A. N. Thomas and W. P. Schecter, "Acute Tracheobronchial Injury,” The Journal of Trauma, Vol. 26, No. 10, 1989, pp. 1320-1330.

[10] M. M. Rossbach, S. B. Johnson, M. A. Gomez, et al., "Management of Major Tracheobronchial Injuries: A 28 Year Experience,” The Annals of Thoracic Surgery, Vol. 65, No. 1, 1998, pp. 182-186. doi:10.1016/S0003-4975(97)01001-1

[11] J. Mordehai, E. Kurtzbart, V. Kapuller, et al., "Tracheal Rupture after Blunt Chest Trauma in a Child,” Journal of Pediatric Surgery, Vol. 32, No. 1, 1997, pp. 104-105. doi:10.1016/S0022-3468(97)90108-1

[12] M. O. Maiwand, K. J. Zehr, C. M. Dyke, et al., “The Role of Cryotherapy for Airway Complications after Lung and Heart-Lung Transplantation,” European Journal CardioThoracic Surgery, Vol. 12, No. 4, 1997, pp. 549-554. doi:10.1016/S1010-7940(97)00208-X 